\title{
Analysis of electrostatic interaction energies in complexes of IFIT5 proteins with RNA via UBDB+EPMM method
}

\author{
Urszula Anna Budniak, Paulina Maria Dominiak \\ Department of Chemistry, University of Warsaw, Warszawa, Poland. \\ urszula.budniak@uw.edu.pl
}

Characterization of electrostatic interactions in selected complexes of IFIT5 proteins with RNA is the aim of my current project. IFITs (Interferon-induced proteins with tetratricopeptide repeats) are effectors of innate immune system, which are expressed in cells infected by viruses. By binding foreign RNA they prevent synthesis of viral proteins in human host cell. IFIT1, IFIT2 and IFIT5 bind different forms of RNA (with triphosphate group or cap at 5' end of RNA). For my study I focused on IFIT5 proteins which are supposed to bind preferentially ppp-RNA.

Usually, the most significant contribution to interaction energy has electrostatic energy. Electrostatic energy can be calculated for large complexes; thus, it is a perfect tool for estimating interaction energy in biomacromolecules. One of the more advanced methods to calculate this energy is University at Buffalo Pseudoatom DataBank (UBDB) together with Exact Potential Multipole Method (EPMM). UBDB enables reconstruction of charge density for macromolecules in quantitative manner. By UBDB+EPMM approach, which takes also charge penetration effects into account, it is possible to compute electrostatic energies with similar accuracy as with quantum chemistry methods.

Calculations of energy are based on the structures of IFIT5 proteins deposited in Protein Data Bank (PDB). I wanted to verify the hypothesis of the lack of influence of RNA sequence on interaction energy in IFIT-RNA complexes investigating three IFIT5pppRNA complexes with different short polynucleotide chains. I examined thoroughly electrostatic interactions and also penetration effect.

Describing the nature of IFIT proteins interaction can help to expand our knowledge about mechanism of selective binding RNA and how human immune system recognizes and destroys viruses.

Keywords: Electrostatic interaction energy, UBDB databank, protein-RNA complexes

Project was financed from the grant PRELUDIUM11 of National Science Centre, Poland nr 2016/21/N/ST4/03722. 